\title{
Generalized parton distributions of the pion in a Bethe-Salpeter approach
}

\author{
S. Noguera因 L. Theuß1 and V. Vent团 \\ Departamento de Física Teòrica and Instituto de Física Corpuscular, \\ Universidad de Valencia - CSIC, E-46100 Burjassot (Valencia), Spain.
}

(Dated: February 8, 2008)

\begin{abstract}
We calculate generalized parton distribution functions in a field theoretic formalism using a covariant Bethe-Salpeter approach for the determination of the bound-state wave function. We describe the procedure in an exact calculation in scalar Electrodynamics proving that the relevant corrections outside our scheme vanish. We extend the formalism to the Nambu-Jona-Lasinio model, a realistic theory of the pion. We go in both cases beyond all previous calculations and discover that all important features required by general physical considerations, like symmetry properties, sum rules and the polynomiality condition, are explicitly verified. We perform a numerical study of their behavior in the weak and strong coupling limits.
\end{abstract}

PACS numbers: 24.10.Jv, 11.10.St, 13.40.Gp, 13.60.Fz

\section{INTRODUCTION}

Hard reactions provide important information for unveiling the structure of hadrons. The large virtuality, $Q^{2}$, involved in these processes allows the factorization of the hard (perturbative) and soft (non-perturbative) contributions in their amplitudes. Therefore these reactions are receiving great attention by the hadronic physics community. Among the hard processes, the Deeply Virtual Compton Scattering (DVCS) merits to be singled out, because it can be expressed, in the asymptotic regime, in terms of the so called generalized parton distributions (GPDs) 1, 2, 3, 4]. The GPDs describe non-forward matrix elements of light-cone operators and therefore measure the response of the internal structure of the hadrons to the probes $[5,6,67,[8]$. There is much effort under way related to the measurement of these functions.

Due to the impossibility at present to determine the GPDs from Quantum Chromodynamics directly, models have been used to provide estimates which should serve to guide future experiments $19,10,11,12,13,14$. The aim of our work is to perform such a calculation in a field theoretic scheme which treats the bound-state in a fully covariant manner following the Bethe-Salpeter approach. In this way we would like to preserve all invariances of the problem. For simplicity we shall use mesons as initial and final states.

We define a scheme, to calculate the electro-magnetic interaction of hadrons, which separates the soft parts, where we use a non perturbative treatment, and the hard parts, where the conventional perturbative treatment is applied. The scheme preserves gauge invariance to leading order and leading twist.

In order to describe the soft part we start by using as a test of our ideas a model based on the $\phi^{4}$ field theory. This theory has the advantage of being renormalizable and therefore any contribution can be analyzed properly. We show, for example, that a correction to the hand bag contribution to first order in the strong coupling constant, which goes beyond our scheme, vanishes.

We next proceed to the main development of this paper, namely to perform the study of the GPDs of the pion by using the Nambu-Jona-Lasinio (NJL) model to describe its structure. The NJL model is not a toy model. In fact, it is the most realistic model for the pion based on a quantum field theory built with quarks. It gives a good description of the low energy physics of the pion and respects the realization of chiral symmetry [15]. Moreover it has been used as the model to tune many coefficients of Chiral Perturbation Theory 16].

The NJL model is a non renormalizable field theory and therefore a cut-off procedure has to be defined. We have chosen the Pauli-Villars regularization procedure because it respects all the symmetries of the problem. In our scheme, we use the NJL model to describe the soft (non perturbative) part of the process, i.e. the initial and final states, while for the hard part we use conventional perturbative QCD. The use of the NJL model allows to calculate the GPDs for massive pions. Some peculiarities, as the non vanishing of the GPDs at the kinematic boundary regions, $x=0$ and $x=1$, well known in the exact $m_{\pi}=0$ limit, survive when $m_{\pi} \neq 0$.

\footnotetext{
*Electronic address: Santiago.Noguera@uv.es

${ }^{\dagger}$ Electronic address: theussl@triumf.ca Present address: TRIUMF, Vancouver, B.C. V6T 2A3, Canada

‡Electronic address: Vicente.Vento@uv.es
} 

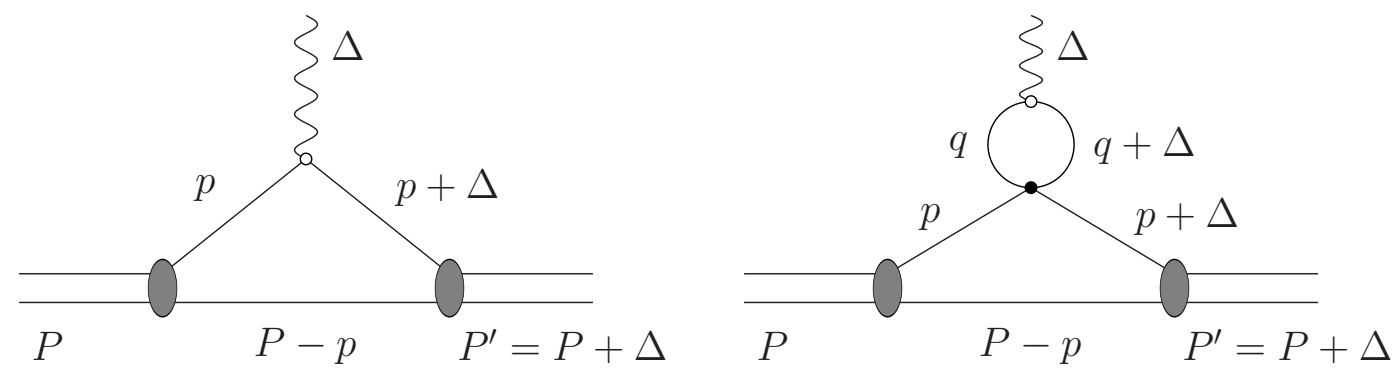

FIG. 1: Diagrams up to order $g$ for the GPDs. The black dot indicates the scalar interaction vertex, while the white blob indicates the effective quark - gauge-boson vertex.

Our paper is organized as follows. In section II we give some general definitions for scalar partons and we introduce our kinematical variables. In section III and IV we will define our approach for the scalar and the NJL model respectively. Section V presents our results and Section VI our conclusions.

\section{GENERALIZED PARTON DISTRIBUTIONS}

The GPDs are non-diagonal matrix elements of bi-local field operators. Various conventions, reference frames, variables, etc., have been used in the literature for the description of such objects. Our notation is represented in Fig. 1 i.e., the initial momentum is labeled by $P$, the final momentum by $P^{\prime}$, and the momentum transfer is given by $\Delta=P^{\prime}-P$. We shall describe initially a model with scalar particles, the generalization to particles with spin is straightforward and will be outlined in a later section. The GPD of a scalar system is defined by the matrix elements of bi-local scalar field operators $1,2,[3,4]$ :

$$
\left.\mathcal{J}^{+} \equiv \frac{1}{2} \int \frac{d z^{-}}{2 \pi} e^{i x P^{+} z^{-}}\left\langle P^{\prime}\left|\Phi^{\dagger}(0) \stackrel{\leftrightarrow}{\partial}+\Phi(z)\right| P\right\rangle\right|_{z^{+}=z^{\perp}=0}=\mathcal{H}(x, \zeta, t)
$$

where $x$ is the conventional Bjorken variable, $\zeta$ the so-called skewedness parameter, and $\overleftrightarrow{\partial}=\vec{\partial}-\overleftarrow{\partial}$. The elastic electromagnetic form factor of a system composed of two scalar particles is given by:

$$
J^{+} \equiv\left\langle P^{\prime}\left|\Phi^{\dagger}(0) \overleftrightarrow{\partial}^{+} \Phi(0)\right| P\right\rangle=\left(P+P^{\prime}\right)^{+} F(t)
$$

It follows directly from these definitions that integrating the GPD over $x$ gives the form factor,

$$
\frac{2}{2-\zeta} \int \mathcal{H}(x, \zeta, t) d x=F(t)
$$

where the dependence on the skewedness parameter $\zeta$ drops out. This result is an important constraint for any model calculation.

Let us now present our notation. Any four vector $v^{\mu}$ will be denoted $\left(v^{+}, v^{\perp}, v^{-}\right)$, where the light cone variables are defined by $v^{ \pm}=\left(v^{0} \pm v^{3}\right) / \sqrt{2}$ and the transverse part $v^{\perp}=\left(v^{1}, v^{2}\right)$. For the kinematics indicated in Fig. 11 we introduce the ratios

$$
x=\frac{p^{+}}{P^{+}}, \quad \zeta=-\frac{\Delta^{+}}{P^{+}}
$$

of plus-components. With these definitions, which differ from other conventional ones [17, 18], both $x$ and $\zeta$ are defined on the interval $[0,1]$.

We are only going to consider elastic processes, so $P^{2}=P^{\prime 2}=M^{2}$ and $\Delta^{2}=t$. The following relation is true in general:

$$
\left(\Delta^{\perp}+\zeta P^{\perp}\right)^{2}=-\zeta^{2} M^{2}-(1-\zeta) t
$$

the positivity of which implies an upper bound for the skewedness $\zeta$ at a given value of the momentum transfer $t$ : $\zeta \leq(-t) /\left(2 M^{2}\right)\left(\sqrt{1+4 M^{2} /(-t)}-1\right) \leq 1$ 


\section{SCALAR ELECTRODYNAMICS}

We begin our study with the simplest model which allows for a completely analytic solution of the Bethe-Salpeter equation: this model describes a bound state of two distinguishable equal-mass scalar particles bound together by a zero-range interaction. For later convenience, we choose only one of the constituent particles to be charged. Our Lagrangian is,

$$
\mathcal{L}=\left[D_{\mu} \phi\right]^{\dagger}\left[D^{\mu} \phi\right]-m^{2} \phi^{\dagger} \phi+\frac{1}{2} \partial_{\mu} \chi \partial^{\mu} \chi-\frac{1}{2} m^{2} \chi^{2}-\frac{g}{2}\left(\phi^{\dagger} \phi \chi^{2}\right)
$$

with $D_{\mu}=\partial_{\mu}+i e A_{\mu}$ so that the electromagnetic charge only couples to the field $\phi$. Assuming that the coupling constant $g$ is larger than a critical value, we have bound states stemming from the last term in Eq. (6). The corresponding Bethe-Salpeter equation is trivially solved in the ladder approximation [19], providing the fully covariant amplitude for the bound state of total mass $M^{2}=P^{2}$ :

$$
\Phi(P, p)=\frac{C}{\left(p^{2}-m^{2}+i \epsilon\right)\left((P-p)^{2}-m^{2}+i \epsilon\right)},
$$

where $p$ is the four-momentum of one of the two constituents with equal mass $m$, and $C$ is a normalization constant. The spectrum for the ground state in this model, can be obtained directly from the Bethe-Salpeter equation, and is given by

$$
1=-i g I_{2}(P)
$$

where the integral $I_{2}(P)$ is given by Eq. (B2). Note that this integral is actually divergent, so some sort of renormalization would be required in order to calculate the spectrum. The theory defined by Eq. (6) is renormalizable and a renormalization program for bound states can be defined. However, for the evaluation of parton distribution functions in this model, we do not encounter any divergent integrals, we shall therefore ignore any matters of renormalization.

The model defined by the above equations is certainly not realistic enough to furnish a reasonable description of real, physical bound states, like the pion for instance. Its main advantage lies in its simplicity, the fact that one may obtain analytic solutions, avoiding approximations that might destroy physical requirements or symmetries, like Lorentz- or Gauge invariance, sum rules, etc. Moreover it has the added advantage that it is defined within a renormalizable quantum field theory. These properties make it a useful playground to perform benchmark calculations, as it was used recently in order to test the viability of certain relativistic quantum mechanics approaches [20, 21].

\section{A. Generalized parton distribution function}

Our starting point is to write the generalized parton distribution (GPD) as an integral over Bethe-Salpeter amplitudes. To do so we could use Eq. (1), a procedure which we shall develop in the NJL case. However for completeness we follow here the procedure of ref. [22, 23], which establishes a relation between the calculation of the GPD and that of the electromagnetic form factor. Namely, in the scattering process $\gamma^{*}+\pi \rightarrow \gamma+\pi$, the leading "hand-bag" diagram, in the deep inelastic limit, reduces to a triangle diagram with an effective vertex (containing two-photon contributions), resulting from the contraction of the propagator with infinite momentum (see first diagram in Fig. 1). Then,

$$
\begin{aligned}
\mathcal{H}(x, \zeta, t) & =-\frac{i}{2} \int \frac{d^{4} p}{(2 \pi)^{4}} \delta\left(p^{+}-x P^{+}\right) \bar{\Phi}(P, p)(\Delta+2 p)^{+}\left[(P-p)^{2}-m^{2}\right] \Phi(P+\Delta, p+\Delta) \\
& =\frac{2 x-\zeta}{2} \frac{1}{i} \int \frac{d^{4} p}{(2 \pi)^{4}} \delta\left(x-p^{+} / P^{+}\right) \bar{\Phi}(P, p)\left[(P-p)^{2}-m^{2}\right] \Phi(P+\Delta, p+\Delta) .
\end{aligned}
$$

Note that the adjoint Bethe-Salpeter amplitude $\bar{\Phi}$ is defined via an anti-chronological time ordering of field operators [19] as compared to $\Phi$, which means that the infinitesimal part $i \epsilon$ in Eq. (7) keeps the same sign as compared to the mass term $m^{2}$.

Using the Bethe-Salpeter amplitude of Eq. (7), we obtain

$$
\mathcal{H}(x, \zeta, t)=\frac{2 x-\zeta}{2} \frac{C^{2}}{i} \int \frac{d^{4} p}{(2 \pi)^{4}} \frac{\delta\left(x-p^{+} / P^{+}\right)}{\left(p^{2}-m^{2}+i \epsilon\right)\left[(p+\Delta)^{2}-m^{2}+i \epsilon\right]\left[(P-p)^{2}-m^{2}+i \epsilon\right]}
$$


Inspecting the pole structure of the integrand for the evaluation of the $p^{-}$integral, we note that it vanishes unless $0 \leq x \leq 1$, i.e., the GPDs have the correct support properties. The integral of Eq. (10) may be calculated explicitly. The analytic result for the GPD is then simply

$$
\mathcal{H}(x, \zeta, t)=\frac{C^{2}}{16 \pi^{2}} \frac{2 x-\zeta}{2} \tilde{I}_{3}(m, x, \zeta, t), \quad 0 \leq x \leq 1
$$

with $\tilde{I}_{3}(m, x, \zeta, t)$ given by Eq. (B13). We observe that our result is explicitly covariant, depending only on $x$, $\zeta$ and $t$. Note that the complete solution is explicitly continuous at $x=\zeta$, however, the derivative at this point is discontinuous. We furthermore encounter a zero at the point $x=\zeta / 2$, which is due to the photon vertex, see Eq. (9). The quark distribution function is given by

$$
q(x) \equiv \mathcal{H}(x, 0,0)=\frac{C^{2}}{16 \pi^{2}} \frac{x(1-x)}{m^{2}-x(1-x) M^{2}} .
$$

The normalization integral may be done analytically and determines the normalization constant $C$.

As it is not a common practice to write GPDs as integrals over Bethe-Salpeter amplitudes, we note that we can write $\mathcal{H}(x, \zeta, t)$ for $x>\zeta$ as the product of light cone wave functions, defined by:

$$
\Psi\left(x, p^{\perp}\right) \equiv \frac{P^{+} \sqrt{x(1-x)}}{i \pi} \int d p^{-} \Phi(P, p)=-\frac{C \sqrt{x(1-x)}}{\left(p^{\perp}-x P^{\perp}\right)^{2}+m^{2}-x(1-x) M^{2}},
$$

which is non-zero only for $0<x<1$. Using some kinematic relations, the GPD for $x>\zeta$ may then be written as

$$
\left.\mathcal{H}(x, \zeta, t)\right|_{x>\zeta}=\frac{1}{2} \frac{2 x-\zeta}{2} \frac{1}{\sqrt{x(x-\zeta)}} \int \frac{d^{2} p^{\perp}}{(2 \pi)^{3}} \Psi^{*}\left(\frac{x-\zeta}{1-\zeta}, p^{\perp}+\frac{1-x}{1-\zeta} \Delta^{\perp}\right) \Psi\left(x, p^{\perp}\right) .
$$

On the other hand, for $x<\zeta$, the GPD may not directly be written as the product of light front wave functions like in Eq. (14), even though we are still able to find analytic solutions for the integral of Eq. (10).

Another conventional way of writing GPDs is via a parameterization in form of double distributions [7]. The relation of these double distributions and the associated D-term with the results of a scalar model similar to the one considered here was recently discussed in ref. [22].

In Fig. 2 we give examples of GPDs in this model for different values of the binding energy and momentum transfers. We distinguish two scenarios: i) the weak binding scenario $M \approx 2 m$ (up to small binding energies of electromagnetic magnitude); ii) the deep binding scenario, which we choose close to the chiral limit, $M=m_{\pi} \approx 140 \mathrm{MeV}$, for reasons which will become natural in our later study of the Nambu-Jona-Lasinio model.

We checked numerically that the sum rule of Eq. (3) is always exactly satisfied. A more stringent test is the so-called polynomiality condition [4], which states that the moments of the GPDs,

$$
\int_{0}^{1} d x x^{N-1} \mathcal{H}(x, \zeta, t) \equiv F_{N}(\zeta, t),
$$

give functions $F_{N}(\zeta, t)$ that are polynomials in $\zeta$ of order not higher than $N$. It is difficult to verify this analytically in the general case, even with the exact solutions that we obtained. Just in the limiting case $M^{2}=0$, the following relation may be shown to hold:

$$
F_{N}(\zeta, 0)=3 \frac{2 N+(N-2) \sum_{i=1}^{N} \zeta^{i}}{N(N+1)(N+2)}
$$

i.e., the polynomiality condition is exactly satisfied at zero momentum transfer. Note also that for $N=1$, Eq. (16) gives the correct normalization of Eq. (3) for any value of $\zeta$. This shows explicitly that the sum rule is indeed independent of $\zeta$ in this case. For $t \neq 0$ and $M^{2} \neq 0$ we have shown, by numerical integration, that the polynomiality condition also holds (see appendix D).

In the small binding limit, $M^{2} \rightarrow 4 m^{2}$, we recover the peaked nature of the GPDs, that was observed in ref. 23], where also an interpretation was given. For small binding energies, the constituents are almost free, so their wave function is highly peaked in momentum space. The two peaks at $x=1 / 2$ and $x=(1+\zeta) / 2$ correspond just to the maxima of the corresponding wave function in the overlap formula Eq. (14). In the exact limit $M=2 m$, the GPDs 

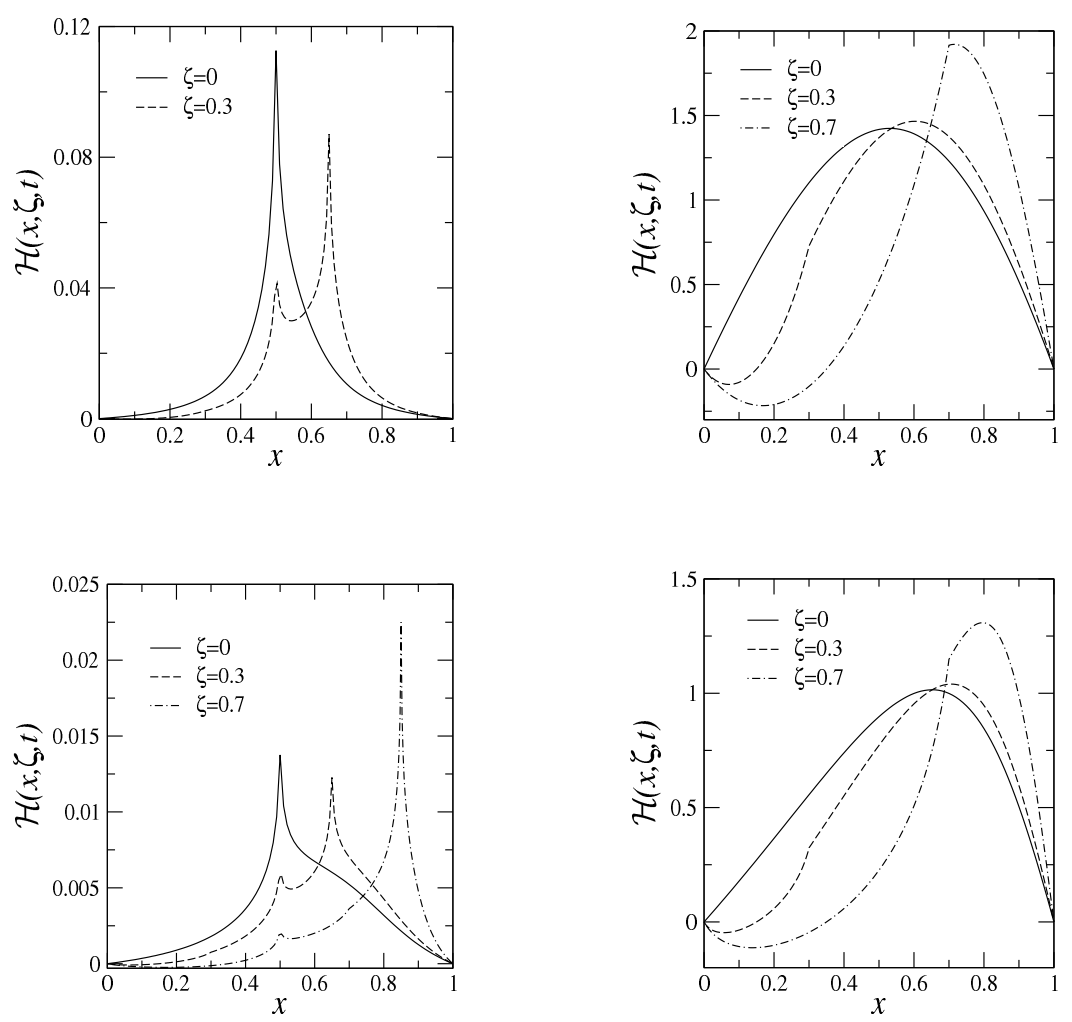

FIG. 2: Generalized parton distributions in the scalar model for different values of the bound state mass $M$ and momentum transfers $t$. The top row gives results for $t=-0.1 \mathrm{GeV}^{2}$. The lower row those for $t=-1 \mathrm{GeV}^{2}$. The graphs follow the scenarios discussed in the text: i) (weak binding, $M \approx 2 m$ ) left column and ii) (deep binding, $M=m_{\pi}$ ) right column. The values of the third variable $\zeta$ are shown in the figures. Note that the sum rule of Eq. (3) is exactly satisfied for each graph.

reduce to a sum of two $\delta$-functions ${ }^{1}$ :

$$
\mathcal{H}(x, \zeta, t)=\frac{1}{2}\left[\delta\left(x-\frac{1}{2}\right)+\delta\left(x-\frac{\zeta+1}{2}\right)\right] \frac{2-\zeta}{2} F(t) .
$$

In particular, the quark distribution function $q(x)=\mathcal{H}(x, 0,0)$ becomes simply $q(x)=\delta(x-1 / 2)$, which means that the particles are free.

In the deep binding limit, $M=m_{\pi}$, the GPDs change from the sharp peaks of the previous case to broad bumps describing the Fermi motion of the deeply bound constituents. At zero momentum transfer, $t=0$, we find the simple form

$$
\mathcal{H}(x, \zeta, 0)=6 \frac{2 x-\zeta}{2}\left\{\frac{x}{\zeta} \theta(\zeta-x) \theta(x)+\frac{1-x}{1-\zeta} \theta(x-\zeta) \theta(1-x)\right\}
$$

\section{B. Electromagnetic form factor}

Calculating the electromagnetic form factor according to Eq. (2), we find:

$$
\left(P+P^{\prime}\right)^{\mu} F\left(Q^{2}\right)=i C^{2} \int \frac{d^{4} p}{(2 \pi)^{4}} \frac{(\Delta+2 p)^{\mu}}{\left(p^{2}-m^{2}+i \epsilon\right)\left[(p+\Delta)^{2}-m^{2}+i \epsilon\right]\left[(P-p)^{2}-m^{2}+i \epsilon\right]},
$$

\footnotetext{
${ }^{1}$ Note that the form factor in this limit is equal to zero everywhere except at $t=0$, where it is 1 .
} 


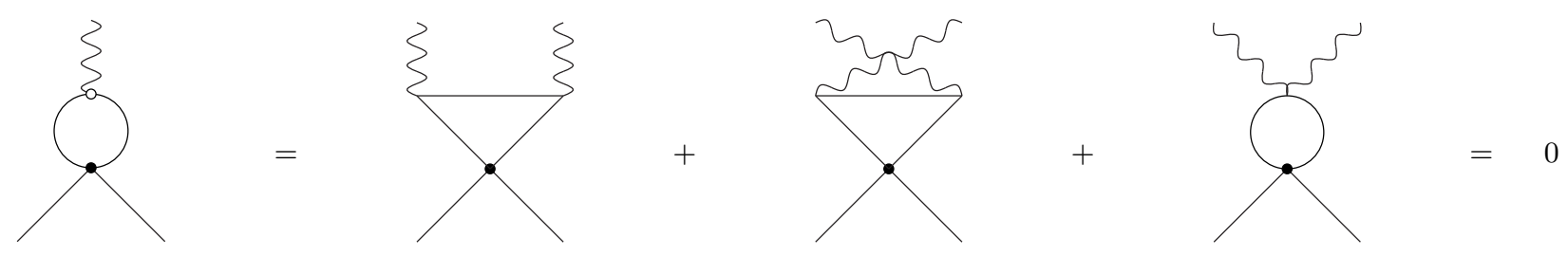

FIG. 3: The second diagram of Fig. 1 contains the loop diagram shown on the left, which corresponds to the expanded set of diagrams on the right, including the seagull term. All of them are divergent, but their sum is not. Moreover when we sum them exactly also there finite parts vanish. The reason behind this cancellation is that the charge is not renormalized to order $g$.

leading to [21],

$$
F\left(Q^{2}\right)=\frac{C^{2}}{16 \pi^{2}} \int_{0}^{1} d z \frac{2}{Q} \frac{1-z}{\sqrt{D}} \log \frac{\sqrt{D}+z Q}{\sqrt{D}-z Q} \equiv \frac{2}{2-\zeta} \int_{0}^{1} d x \mathcal{H}(x, \zeta, t)
$$

with $D=4\left[m^{2}-z(1-z) M^{2}\right]+z^{2} Q^{2}$. This suggests that one could calculate the GPD by a simple change of the integration variable in the expression for the form factor. The general character of this change of variable would not be clear, however. Naturally, at $Q^{2}=0$ we reproduce the normalization condition.

Taking the parameters $m=241 \mathrm{MeV}$ and $M=139 \mathrm{MeV}$, we can evaluate the root-mean squared radius of a "pion" built of scalar particles, via $\left\langle r_{\pi}\right\rangle^{2}=-6 \partial F\left(Q^{2}\right) /\left.\partial Q^{2}\right|_{Q^{2}=0}=(0.47 \mathrm{fm})^{2}$, to be compared to the experimental value of $(0.66 \mathrm{fm})^{2}$. In the limit of a mass-less bound state, $M=0$, we obtain the analytic result $\left\langle r_{\pi}\right\rangle^{2}=3 /\left(10 \mathrm{~m}^{2}\right)=$ $(0.45 \mathrm{fm})^{2}$.

\section{Bubble diagram and Gauge invariance}

Up to first order in the strong coupling constant $g$, we have to calculate the second Feynman diagram of Fig. 1] In scalar Electrodynamics, the second diagram does not contribute to the electromagnetic form factor. The loop integral is proportional to

$$
\int \frac{d^{4} q}{(2 \pi)^{4}} \frac{(2 q+\Delta)^{\mu}}{\left(q^{2}-m^{2}+i \epsilon\right)\left((q+\Delta)^{2}-m^{2}+i \epsilon\right)}
$$

which by virtue of Eq. (B4) is zero. However, this diagram could contribute to the GPDs, because here we keep the + component of the integration variable fixed. In a naive way we observe that the bubble present in the diagram will lead to divergent contributions to the GPDs. In order to understand properly the problem let us consider the DVCS on the pion. Unfolding the procedure introduced in this section, we note that the bubble corresponds to a triangle in which one of the propagators has been contracted due to the infinite momentum carried by the parton. Divergences appear in the calculation and correspond in practice to terms proportional to $\log \left(Q^{2}\right)$ in the triangle diagram. Considering all contributions to the same order (see Fig. Bi), we find that the divergences cancel. But not only the divergent terms cancel, also the finite parts do, therefore the whole contribution, contrary to expectations, vanishes exactly for the GPDs.

It is not difficult to advance a physical argument explaining this cancellation. The diagrams of Fig. 3 can be interpreted as a renormalization of the bare charge present in the seagull term. Due to gauge symmetry this charge must be the square of the charge associated to the one photon vertex, before and after renormalization. But we have seen from Eq. (21) that there is no renormalization (finite or infinite) at order $g$ to the charge. Consequently the sum of diagrams of Fig. 3, or the second diagram of Fig. 1] must vanish.

\section{THE MODEL OF NAMBU AND JONA-LASINIO}

The model of the last section can certainly not provide a very realistic description of electromagnetic properties of a pion because of the scalar nature of the constituents involved. We are therefore going to consider a model with spinor particles. An evident choice to investigate is the model of Nambu and Jona-Lasinio (NJL) [15, 24, 25, 26]. 
This model is the most realistic model for pions built of quarks that is based on a quantum field theory. It gives a good description of the low energy physics of the pion exhibiting the phenomena of dynamical mass generation and spontaneous breaking of chiral symmetry, which are crucial ingredients for low-energy hadronic physics [15, 16].

We start from the Lagrangian density in the two-flavor version of this model where we add the electromagnetic interaction in the usual way,

$$
\mathcal{L}=\bar{\psi}\left(i \not D-\mu_{0}\right) \psi+g\left[(\bar{\psi} \psi)^{2}-\left(\bar{\psi} \vec{\tau} \gamma_{5} \psi\right)^{2}\right]
$$

with $D_{\mu}=\partial_{\mu}+i e A_{\mu}$. Again, assuming the contact interaction of the last term to be mainly responsible for the binding, the Bethe-Salpeter equation in ladder approximation to be fulfilled by a bound state in this model is given by (the factor 2 comes from the symmetry of the interaction):

$$
S^{-1}(p) \vec{\Phi}(P, p) S^{-1}(p-P)=i g \vec{\tau} \gamma_{5} \int 2 \operatorname{Tr}\left\{\vec{\tau} \gamma_{5} \cdot \vec{\Phi}\left(P, p^{\prime}\right)\right\} \frac{d^{4} p^{\prime}}{(2 \pi)^{4}}
$$

Here, the symbol $\operatorname{Tr}$ refers to traces on spinor, flavor and color indices, and $S(p)$ is the single quark propagator

$$
S(p)=\frac{i}{\not p-m+i \epsilon}=i \frac{\not p+m}{p^{2}-m^{2}+i \epsilon}
$$

of a quark with constituent mass $m$, which is generated from the bare mass $\mu_{0}$ via the gap equation Eq. (B9]) [15, 26]. The quantity $\Phi(P, p)$ is the momentum space image of the Bethe-Salpeter amplitude of a bound state with total four-momentum $P$, while $p$ is the four-momentum of one of the constituents. The solution of Eq. (23) is rather trivial since the integral on the right hand side is just a constant, so we can write

$$
\vec{\Phi}(P, p)=i g_{\pi q q} S(p) \vec{\tau} \gamma_{5} S(p-P)=-i g_{\pi q q} \frac{(\not p+m) \vec{\tau} \gamma_{5}(\not p-\not p+m)}{\left(p^{2}-m^{2}+i \epsilon\right)\left[(P-p)^{2}-m^{2}+i \epsilon\right]}
$$

where $g_{\pi q q}$ is the quark-pion coupling constant (given in Eq. (B11) which can be determined from the usual BetheSalpeter normalization condition [15]. Reinserting this solution into Eq. (23) gives a self-consistency condition, (given in Eq. (B10) $)$, which determines the mass of the ground state as a function of the coupling constant. Note that in the chiral limit, when $P^{2}=0$ and $\mu_{0}=0$, Eq. (B10) is nothing but the gap equation (B9), providing some evidence for the self-consistency of the procedure.

The NJL model has been investigated quite extensively in different domains of physics with rather impressive success (see refs. 15, 26] for reviews), it is therefore natural to test its predictions for GPDs of the pion. Similar studies have been carried out recently [7, 27, 28, 29, 30] with different models. One drawback of the NJL model is of course its non-renormalizability, which makes it useful only as an effective, low-energy model that, however, may be regarded as a non-linear realization of the QCD Lagrangian. Numerical results therefore usually depend on the regularization scheme employed to deal with the divergent integrals. As thoroughly discussed in refs. [31, 32], a suitable regularization method has to satisfy a certain number of requirements. The method that was found to be most suitable was a Pauli-Villars with two subtractions, this is the one that we shall adopt, as outlined in appendix $\mathrm{A}$

\section{A. Generalized parton distribution}

We are interested in calculating the diagrams of Fig. 4 in the Nambu-Jona-Lasinio Model. A general expression for the matrix element of the bi-local $u$-quark current in the Bethe-Salpeter approach is

$$
\begin{aligned}
&\left\langle P^{\prime}\left|\bar{\Psi}_{u}\left(x^{\prime}\right) \gamma_{\mu} \Psi_{u}(x)\right| P\right\rangle=\int d^{4} x_{2} \operatorname{Tr}\left\{\bar{\Phi}_{P^{\prime}}^{i}\left(x^{\prime}, x_{2}\right) \frac{1}{2}\left(1+\tau_{3}\right) \gamma_{\mu}\left[\Phi_{P}^{i}\left(x, x_{2}\right)\left(i \not \not^{(2)}-m_{2}\right)\right]\right\} \\
&+\int d^{4} x_{1} \operatorname{Tr}\left\{\bar{\Phi}_{P^{\prime}}^{i}\left(x_{1}, x\right)\left(i \not \vec{\not}^{(1)}-m_{1}\right) \Phi_{P}^{i}\left(x_{1}, x^{\prime}\right) \frac{1}{2}\left(1+\tau_{3}\right) \gamma_{\mu}\right\},
\end{aligned}
$$

where $i$ is the isospin index. The first (second) term in this expression corresponds to the contribution of the first (second) constituent of the system, and indices 1 and 2 refer to coordinates or operators related to particles 1 and 2 , bound in the meson. In terms of momentum variables we have

$$
\vec{\Phi}_{P}\left(x_{1}, x_{2}\right)=e^{-i P \cdot X} \int \frac{d^{4} k}{(2 \pi)^{4}} e^{-i k r} \vec{\Phi}(k, P),
$$



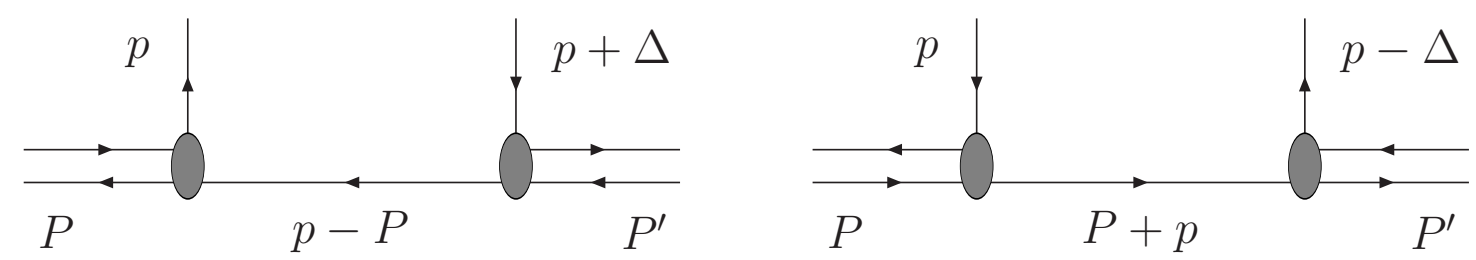

FIG. 4: Diagrams contributing to the GPD in the Nambu-Jona-Lasinio Model.

with the center-of-mass and relative coordinates defined by

$$
X=\mu_{1} x_{1}+\mu_{2} x_{2}, \quad r=x_{1}-x_{2}, \quad \mu_{1,2}=\frac{m_{1,2}}{m_{1}+m_{2}},
$$

and $P=p_{1}+p_{2}, k=\mu_{2} p_{1}-\mu_{1} p_{2}$ the total and relative four-momentum of the system.

We shall concentrate on the $u$-quark parton distribution in a $\pi^{+}$meson, for which we have to consider the first diagram of Fig. 4 with $p_{1}=p, p_{2}=P-p$. The second diagram of Fig. 4 contributes to the $d$-quark parton distribution with $p_{1}=-p, p_{2}=P+p$. Inserting Eq. (27) and taking $m_{1}=m_{2}=m$, the diagrams of Fig. 4 give the following contribution to the GPDs:

$$
\begin{aligned}
& \left.\frac{1}{2} \int \frac{d z^{-}}{2 \pi} e^{i x P^{+} z^{-}}\left\langle P^{\prime}\left|\bar{\Psi}_{u}(0) \gamma^{+} \Psi_{u}(z)\right| P\right\rangle\right|_{z^{+}=z^{\perp}=0}=\mathcal{H}(x, \zeta, t)= \\
& =\frac{1}{2} \int \frac{d^{4} p}{(2 \pi)^{4}} \delta\left(x-p^{+} / P^{+}\right) \operatorname{Tr}\left\{\bar{\Phi}^{\pi^{+}}\left(p+P^{\prime}-P, P^{\prime}\right) \frac{1}{2}\left(1+\tau_{3}\right) \gamma^{+} \Phi^{\pi^{+}}(p, P)(\not p-\not p-m)\right\},
\end{aligned}
$$

where we have switched back to particle momenta. With the Bethe-Salpeter amplitude of Eq. (25) we obtain an expression for the GPD in the Nambu-Jona-Lasinio model that is similar to Eq. 10):

$$
\mathcal{H}(x, \zeta, t)=i 4 N_{c} g_{\pi q q}^{2} \int \frac{d^{4} p}{(2 \pi)^{4}} \delta\left(x-p^{+} / P^{+}\right) \frac{(x+1-\zeta)\left(p^{2}-m^{2}\right)+p \cdot \Delta-x P \cdot \Delta-(2 x-\zeta) p \cdot P}{\left(p^{2}-m^{2}+i \epsilon\right)\left[(p+\Delta)^{2}-m^{2}+i \epsilon\right]\left[(P-p)^{2}-m^{2}+i \epsilon\right]} .
$$

The $p^{-}$integral in Eq. (30) is evaluated by the usual residue calculus. Due to the pole structure of the integrand we obtain two contributions, the first one in the region $\zeta<x<1$, corresponding to the quark contribution and the second in the region $0<x<\zeta$, corresponding to a quark-anti-quark contribution. The second diagram of Fig. 4 will give an anti-quark contribution in the region $\zeta-1<x<0$, and a quark-antiquark contribution in the region $0<x<\zeta$, just like the first diagram. In case of a $\pi^{+}$meson, the first diagram gives the $u$-quark distribution, while the second one gives the $\bar{d}$-quark distribution, but due to isospin symmetry, both distributions are related by $\mathcal{H}_{u}(x, \zeta, t)=-\mathcal{H}_{\bar{d}}(\zeta-x, \zeta, t)$. Concentrating only on the $u$-quark distribution and employing a Pauli-Villars regularization, we get for $\zeta<x<1$ :

$$
\begin{aligned}
\left.\mathcal{H}(x, \zeta, t)\right|_{\zeta<x<1}=\frac{N_{c} g_{\pi q q}^{2}}{4 \pi^{2}} \sum_{j=0}^{2} c_{j}\left\{-\log \frac{m_{j}^{2}}{m^{2}}-\frac{1}{2} \log \frac{m_{j}^{2}-\bar{x} M^{2}}{m_{j}^{2}}-\frac{1}{2} \log \frac{m_{j}^{2}-\bar{y} M^{2}}{m_{j}^{2}}\right. \\
\left.+\frac{(2 x-\zeta) M^{2}+(1-x) t}{2} \tilde{I}_{3}\left(m_{j}, x, \zeta, t\right)\right\},
\end{aligned}
$$

with the abbreviations $\bar{x}=x(1-x), \bar{y}=(1-x)(x-\zeta) /(1-\zeta)^{2}$, while $\tilde{I}_{3}(m, x, \zeta, t)$ is given by Eq. (B13) with $m=m_{0}$.

Turning our attention to the non-valence region, $0<x<\zeta$, we find a first contribution arising from the diagram

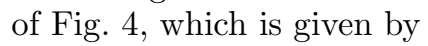

$$
\begin{aligned}
\left.\mathcal{H}^{a}(x, \zeta, t)\right|_{0<x<\zeta}=\frac{N_{c} g_{\pi q q}^{2}}{4 \pi^{2}} \sum_{j=0}^{2} c_{j}\left\{-\frac{x}{\zeta} \log \frac{m_{j}^{2}}{m^{2}}-\frac{1}{2} \log \frac{m_{j}^{2}-\bar{x} M^{2}}{m_{j}^{2}}-\frac{2 x / \zeta-1}{2} \log \frac{m_{j}^{2}-\bar{y} t}{m_{j}^{2}}\right. \\
\left.+\frac{(2 x-\zeta) M^{2}+(1-x) t}{2} \tilde{I}_{3}\left(m_{j}, x, \zeta, t\right)\right\},
\end{aligned}
$$



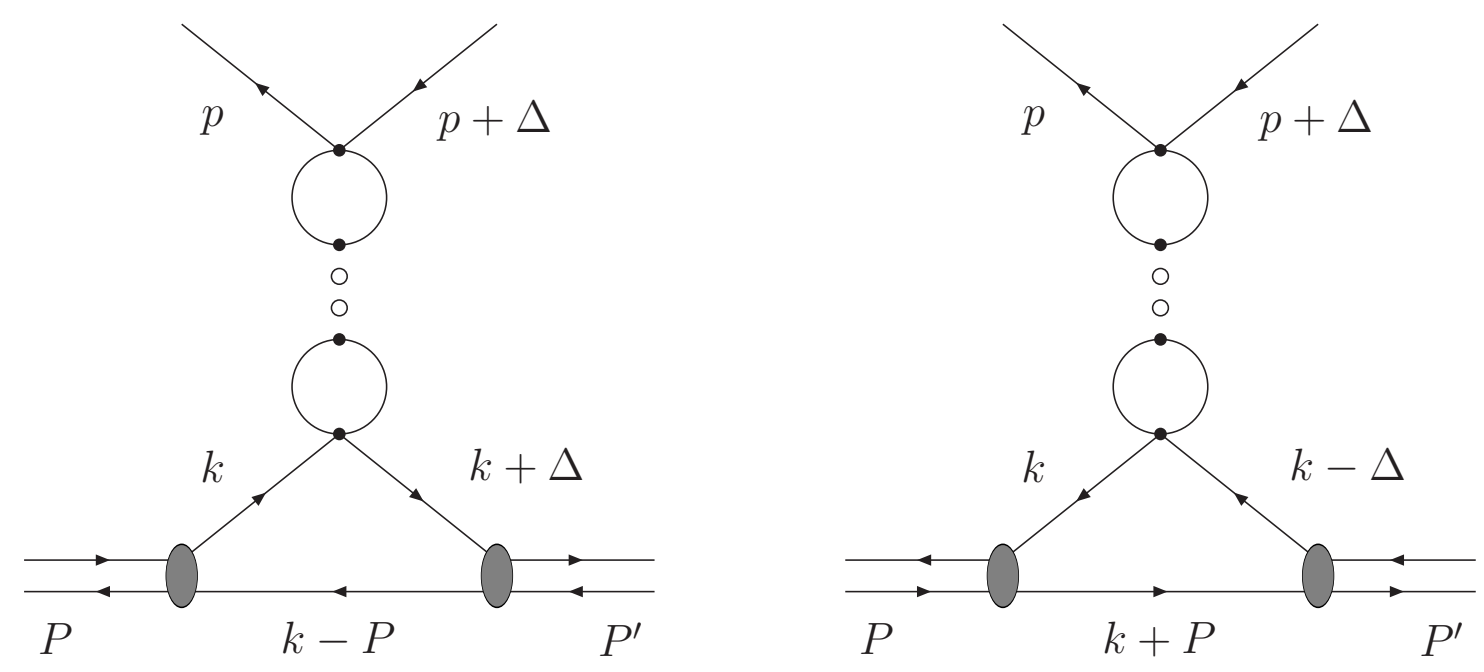

FIG. 5: Diagrams contributing to the GPDs in the Nambu-Jona-Lasinio model coming from the " $\sigma$ " coupling to the two photons. They contribute in the region $0<x<\zeta$.

where now $\bar{y}=x(\zeta-x) / \zeta^{2}$. In the region $0<x<\zeta$, there is a second contribution coming from the re-scattering diagrams of Fig. [5. They correspond to the coupling of the two photons in a channel with the quantum numbers of

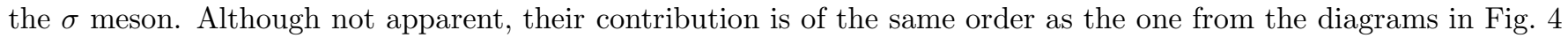
(see appendix $\mathbb{C}$ for details of the calculation). The result for the sum of these two diagrams is

$$
\left.\mathcal{H}^{b}(x, \zeta, t)\right|_{0<x<\zeta}=\frac{N_{c} g_{\pi q q}^{2}}{4 \pi^{2}}\left(1-\frac{2 x}{\zeta}\right) C(t) \sum_{j=0}^{2} c_{j}\left[-\ln \frac{m_{j}^{2}}{m^{2}}-\ln \frac{m_{j}^{2}-t \bar{y}}{m_{j}^{2}}\right],
$$

with

$$
C(t)=m^{2} \frac{\left[\left(t-2 M^{2}\right) I_{3}\left(P, P^{\prime}\right)+2 I_{2}\left(P-P^{\prime}\right)\right]}{M^{2} I_{2}\left(M^{2}\right)+\left(4 m^{2}-t\right) I_{2}\left(P-P^{\prime}\right)}
$$

and $\bar{y}=x(\zeta-x) / \zeta^{2}$.

In the exact chiral limit, $M=m_{\pi}=0$ and $m_{\sigma}=2 m$, Eq. (34) becomes

$$
C(t)=\frac{m^{2}}{\left(4 m^{2}-t\right)}\left[2+t \frac{I_{3}\left(P, P^{\prime}\right)}{I_{2}\left(P-P^{\prime}\right)}\right]
$$

where the $\sigma$ propagator appears in an explicit way. This propagator is also present in Eq. (34), but it is not so apparent due to the more complicated structure of the equation. Thus we can speak effectively of a coupling of the two photons to the $\sigma$ state, and this contribution is a requirement of chiral symmetry.

The total value for the GPD in the region $0<x<\zeta$ is obtained by summing the two,

$$
\left.\mathcal{H}(x, \zeta, t)\right|_{0<x<\zeta}=\left.\mathcal{H}^{a}(x, \zeta, t)\right|_{0<x<\zeta}+\left.\mathcal{H}^{b}(x, \zeta, t)\right|_{0<x<\zeta} .
$$

We note that the first three terms in curly brackets of Eq. (31) give contributions that are independent of the momentum transfer $t$. The first term, in particular, gives an overall constant, independent of $x, \zeta$ and $t$, which does not vanish for $x=1$. From Eq. (31) we get the distribution function at zero momentum transfer,

$$
q(x)=\mathcal{H}(x, 0,0)=\frac{N_{c} g_{\pi q q}^{2}}{4 \pi^{2}} \sum_{j=0}^{2} c_{j}\left\{-\log \frac{m_{j}^{2}}{m^{2}}-\log \frac{m_{j}^{2}-\bar{x} M^{2}}{m_{j}^{2}}+\frac{m_{j}^{2}}{m_{j}^{2}-\bar{x} M^{2}}\right\}
$$

which is consistent with the normalization condition.

In Fig. [6] we give some examples of GPDs in the NJL model for different values of the binding energy and momentum transfers. We distinguish in here three scenarios: i) the weak binding scenario $M \approx 2 m$ (up to small binding energies 
of electromagnetic magnitude) ; ii) the deep binding scenario, with binding energies in the tens of $\mathrm{MeV}$ range, which would correspond physically to a strongly bound state, i.e. like the $\rho$ meson; iii) the Goldstone boson scenario, which we take close to the chiral limit, $M=m_{\pi} \approx 140 \mathrm{MeV}$. This classification is very natural as the behavior of the GPDs in the figures shows. The Goldstone boson scenario, with its flat GPDs, did not appear in scalar Electrodynamics and it shows how spontaneous chiral symmetry breaking appears in the GPDs.

For zero binding, there is no spontaneous symmetry breaking, thus no pion, and the constituent quarks are quasi free. For weak binding, we expect for the GPDs a similar behavior to the one discussed in scalar Electrodynamics. This similarity can be seen by comparing the corresponding graphs of Figs. 2 and 6 In the weak binding limit we note again the appearance of two peaks at $x=1 / 2$ and $x=(1+\zeta) / 2$, which go over into $\delta$-functions in the exact limit $M=2 m$, just as in the scalar case, see Eq. (17). As soon as there is binding the GPDs differ more and more from the free ones as we approach the chiral $M=m_{\pi}$ limit with massive constituent quarks.

A few properties of the final result may be recognized from the analytic expressions of the GPDs. First, the total GPDs are discontinuous at $x=\zeta$. The discontinuity arises from the diagrams in Fig. 5 since the contribution from the diagrams in Fig. [ 4 to the GPDs is continuous, even though the derivative at $x=\zeta$ is not. Second, we see that $\mathcal{H}(0, \zeta, t) \neq 0$ for any value of $\zeta$. Its value, which depends on $t$ and $M$ but not on $\zeta$, is connected to the discontinuity by:

$$
\mathcal{H}(0, \zeta, t)=\mathcal{H}\left(\zeta_{+}, \zeta, t\right)-\mathcal{H}\left(\zeta_{-}, \zeta, t\right) \neq 0
$$

Third, as noted above, $\mathcal{H}(1, \zeta, t) \neq 0$. Its value is independent of $t$ and $\zeta$, and only depends on the bound state mass $M$ via the coupling constant $g_{\pi q q}$. In particular, in the chiral limit, when $M=0$, the value of $\mathcal{H}(x, \zeta, t)$ at $x=1$ is simply given by:

$$
\mathcal{H}(1, \zeta, t)=1 .
$$

Furthermore, the distribution functions become relatively more concentrated around $x=1$ for large $|t|$, which is just a consequence of the constant value of the distribution function at $x=1$. Finally, in spite of these peculiarities, we find again that Eq. (31) is always exactly satisfied, as we checked numerically.

The polynomiality condition in this case takes a very simple form in the chiral limit. When $M=0$, the following relation holds:

$$
F_{N}(\zeta, 0)=\frac{1}{N}\left(1-\frac{1}{2} \zeta^{N}\right)
$$

i.e., at zero momentum transfer, the polynomiality condition is fulfilled. Again, for $N=1$, Eq. (40) reproduces Eq. (3) in the case $t=0$. For $t \neq 0$ and $M^{2} \neq 0$ we have shown, by numerical integration, that the polynomiality condition also holds. Further details are given in appendix D

The fact that the polynomiality condition holds both for SED and NJL in the present scheme should not be a surprise since both models arise from a field theoretic description which preserves Lorentz symmetry, parity, timereversal invariance and hermiticity [4].

Finally we note that in the chiral limit, $M=0$ and at zero momentum transfer, $t=0$, we reproduce the analytic results of ref. [7], namely:

$$
\mathcal{H}(x, \zeta, 0)=\frac{1}{2} \theta(\zeta-x) \theta(x)+\theta(x-\zeta) \theta(1-x),
$$

so in particular, $\mathcal{H}(x, 1,0)=1 / 2$ and $\mathcal{H}(x, 0,0)=1$. It may be checked from Eq. (30) that this result is completely independent of any regularization procedure. This form may be compared with the corresponding result in the scalar model, see Eq. (18). Eq. (41) also implies that in the chiral limit we get a quark distribution function that is equal to unity. This coincides with the results obtained in refs. [32, 33, 34].

\section{B. Isospin decomposition}

Following the idea of ref. [35] we introduce an isospin decomposition for the GPDs of the pion ${ }^{2}$

$$
\left.\int \frac{d z^{-}}{2 \pi} e^{i x P^{+} z^{-}}\left\langle\pi^{a}\left(P^{\prime}\right)\left|\bar{\Psi}_{f^{\prime}}(0) \gamma^{+} \Psi_{f}(z)\right| \pi^{b}(P)\right\rangle\right|_{z^{+}=z^{\perp}=0}=\delta^{a b} \delta_{f f^{\prime}} \mathcal{H}^{I=0}(x, \zeta, t)+\varepsilon^{a b c} \tau_{f f^{\prime}}^{c} \mathcal{H}^{I=1}(x, \zeta, t) .
$$

\footnotetext{
${ }^{2}$ Note that our notation is slightly different from the cited reference.
} 

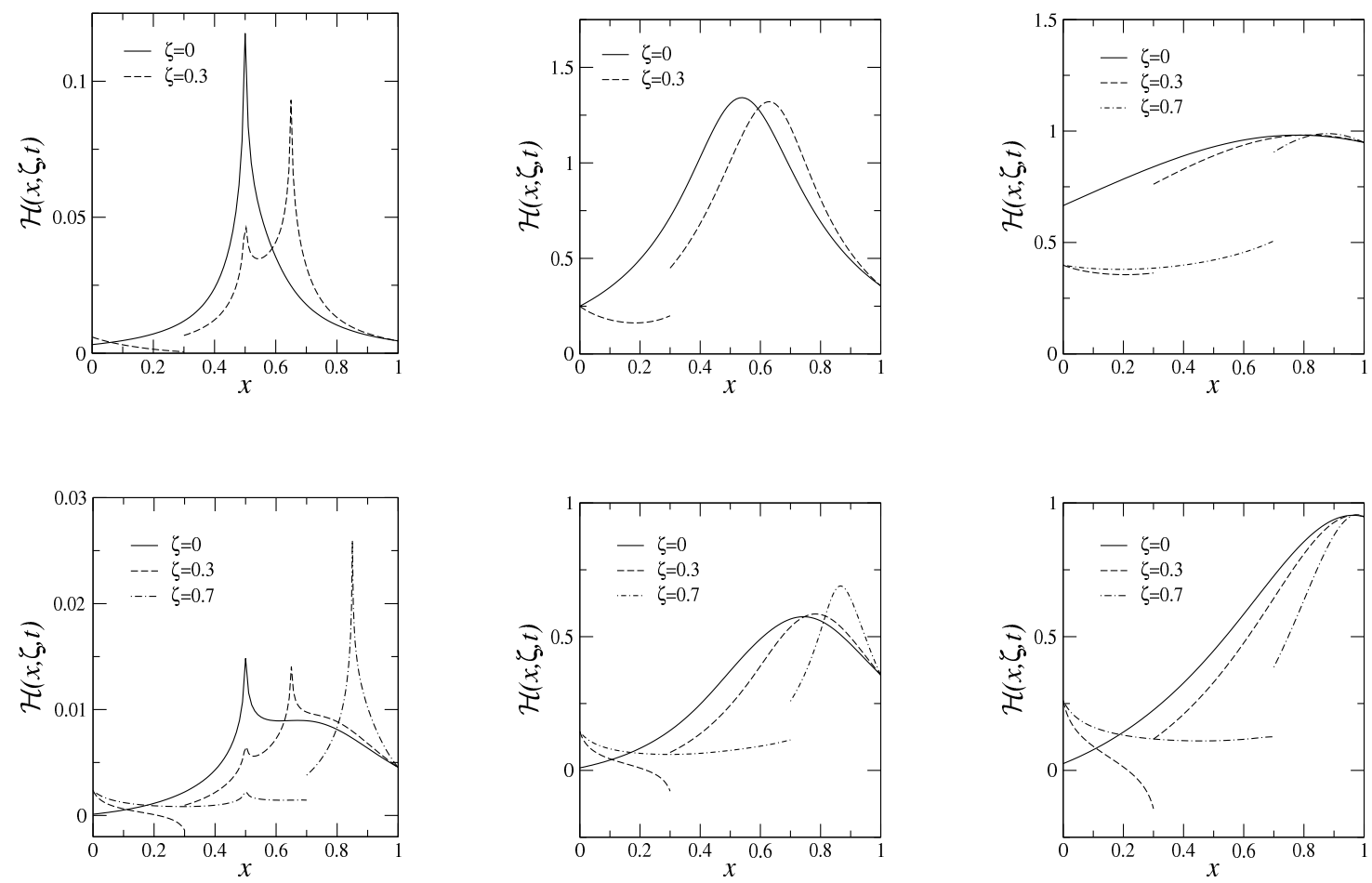

FIG. 6: Generalized parton distributions in the Nambu-Jona-Lasinio model for different values of the bound state mass $M$ and momentum transfers $t$. The top row gives results for $t=-0.1 \mathrm{GeV}^{2}$, while on the bottom for $t=-1 \mathrm{GeV}^{2}$. We describe from left to right the scenarios discussed in the text, i.e., from weak binding $(M \approx 2 m$, left column), to strong binding (middle column) to the chiral limit $(M=0$, right column). Note that the sum rule of Eq. (3) is exactly satisfied for each graph.

In order to extract the two isospin components we need to calculate the $u$-quark GPD for the $\pi^{0}$. We have contributions coming from both diagrams of Fig. 团and from those of Fig. [5] leading to

$$
\mathcal{H}_{u}^{\pi^{0}}(x, \zeta, t)=\frac{1}{2}[\mathcal{H}(x, \zeta, t)-\mathcal{H}(\zeta-x, \zeta, t)],
$$

where $\mathcal{H}(x, \zeta, t)$ is the $u$-quark GPD for the $\pi^{+}$defined in Eqs. (31) and (36). Here $x$ ranges from $\zeta-1$ to 1 , as has been explained below Eq. (30). The GPDs of the $\pi^{+}$and $\pi^{0}$ allow to extract the isospin components

$$
\begin{aligned}
& \mathcal{H}^{I=0}(x, \zeta, t)=2 \mathcal{H}_{u}^{\pi^{0}}(x, \zeta, t) \quad=\mathcal{H}(x, \zeta, t)-\mathcal{H}(\zeta-x, \zeta, t) \\
& \mathcal{H}^{I=1}(x, \zeta, t)=2 \mathcal{H}^{\pi^{+}}(x, \zeta, t)-2 \mathcal{H}_{u}^{\pi^{0}}(x, \zeta, t)=\mathcal{H}(x, \zeta, t)+\mathcal{H}(\zeta-x, \zeta, t) .
\end{aligned}
$$

Using Eq. (411) we obtain simple expressions for the two isospin GPDs in the $t=0, m_{\pi}=0$ case:

$$
\mathcal{H}^{I=0}(x, \zeta, t)=\left\{\begin{array}{rr}
-1, & \zeta-1<x<0 \\
0, & 0<x<\zeta \\
1, & \zeta<x<1
\end{array} \quad ; \quad \mathcal{H}^{I=1}(x, \zeta, t)=1, \quad \zeta-1<x<1 .\right.
$$

We observe that the predictions of ref. [35], regarding the isospin components in the limit $m_{\pi}=0$, are satisfied. The relation between $\mathcal{H}^{I=1}(x, \zeta=1, t=0)$ and the wave function of the pion, $\varphi_{\pi}(x)$, and $\mathcal{H}^{I=0}(x, \zeta=1, t=0)=0$ hold. Note moreover that our pion wave function, in this limit, is $\varphi_{\pi}(x)=1$. The $m_{\pi}=0, \zeta=1$ and $t=0$ case corresponds to a process in which the initial and final pion momenta are $P=\left(P^{+}, \overrightarrow{0}^{\perp}, 0\right)$ and $P^{\prime}=\left(0, \overrightarrow{0}^{\perp}, 0\right)$, respectively. For zero pion mass this process is allowed only as a limit. In the NJL model we can look for deviations from the exact chiral limit.

\section{Electromagnetic form factor}

In order to understand better the above results, we may want to calculate the electromagnetic form factor in the NJL model which is given by the $x$-integrated parton distribution, see Eq. (3). This has been done already in several 
works [15, 36, 37], but we want to investigate the relation with the present results. Calculating again the current we find, from the first diagram of Fig. 11 for the form factor and for the GPDs:

$$
\left(P+P^{\prime}\right)^{\mu} F\left(Q^{2}\right)=8 i N_{c} g_{\pi q q}^{2} \int \frac{d^{4} p}{(2 \pi)^{4}} \frac{\left(p^{\mu}+P^{\mu}+\Delta^{\mu}\right)\left(p^{2}-m^{2}+i \epsilon\right)+p \cdot \Delta P^{\mu}-P \cdot \Delta p^{\mu}-\left(2 p^{\mu}+\Delta^{\mu}\right) p \cdot P}{\left(p^{2}-m^{2}+i \epsilon\right)\left[(p+\Delta)^{2}-m^{2}+i \epsilon\right]\left[(P-p)^{2}-m^{2}+i \epsilon\right]}
$$

Rewriting the denominator under the integral and using some relations of appendix B] we arrive at

$$
F\left(Q^{2}\right)=i 2 N_{c} g_{\pi q q}^{2} \frac{\left(Q^{2} / 2+M^{2}\right) I_{2}(\Delta)+M^{2} I_{2}(P)-M^{4} I_{3}(\Delta,-P)}{Q^{2} / 4+M^{2}} .
$$

We checked numerically (by applying the same Pauli-Villars regularization method) that this form factor is exactly reproduced by integrating our result for the GPD over $x$. This means that the non-vanishing distribution functions (at $x=1$ ) are implicitly present in the result of Eq. (47). Note that in the chiral limit, $M^{2}=0$, we simply have

$$
F\left(Q^{2}\right)=4 i N_{c} g_{\pi q q}^{2} I_{2}(\Delta)=1-R\left(Q^{2}\right) / I_{2}(0)
$$

where for small $Q^{2}, R\left(Q^{2}\right)$ is given by

$$
R\left(Q^{2}\right)=\frac{i}{16 \pi^{2}} \frac{Q^{2}}{6 m^{2}}
$$

so together with the relation $f_{\pi}^{2}=-12 \mathrm{im}^{2} I_{2}(0)$, we can evaluate the root-mean squared radius of the pion in this model via $\left\langle r_{\pi}\right\rangle^{2}=-6 \partial F\left(Q^{2}\right) /\left.\partial Q^{2}\right|_{Q^{2}=0}=3 f_{\pi}^{-2} /\left(4 \pi^{2}\right)$, which is the same as in ref. [15]. With $f_{\pi}=93 \mathrm{MeV}$, we find

the numerical value of $\left\langle r_{\pi}\right\rangle^{2}=(0.585 \mathrm{fm})^{2}$, to be compared to the experimental value of $(0.66 \mathrm{fm})^{2}$. Note, however, that Eq. (49) is valid only in the limit when the cut-off goes to infinity, for the finite cut-off as specified in appendix A the root mean squared radius gets multiplied by a factor $\sim 0.89$.

\section{DISCUSSION}

The results obtained in the scalar Electrodynamics model show a perfect realization of all wishful ingredients. The calculation is exact, finite and satisfies all the desired properties, i.e., the GPDs have the correct support and vanish at the boundary regions, while the sum rule and the polynomiality condition are exactly verified. From the physical point of view, the GPDs show a realization in terms of quasi-free constituents in the weak binding limit. As the binding increases one is confronted with the dynamics as derivable from a non trivial momentum distribution determined by the corresponding Bethe-Salpeter amplitude, a feature also appearing in other model calculations [14]. The physical effect associated with $t$ and $\zeta$ is naturally represented in the GPDs. The variable $t$ tends to push the constituent distribution towards higher values of $x$, which corresponds to an input of momentum transfer into the system, while the variable $\zeta$ incorporates the description of virtual pairs. Unfortunately this model is not very realistic for the pion (perhaps it might be better fit for a description of the nucleon) and our results represent only qualitative features of how the dynamics might influence the distributions.

An equivalent model was considered in refs. [23, 38], but the emphasis was put on the light-front quantization method. The results were calculated only for $x>\zeta$, while the region $x<\zeta$ was explored by an analytic continuation of the vertex function. Due to the approximations used in their approach the continuation is not perfect and therefore sum rules like the one appearing in Eq. (3) are explicitly violated.

For the NJL model the calculation requires regularization. The latter certainly influences the results, as has been discussed in ref. [31], where the Pauli-Villars method was compared to the one of Brodsky-Lepage with different results. The Pauli-Villars method is compatible with all the symmetry requirements [33], which is the reason for our choice. A caveat that should be emphasized is that the model does not only contain the dynamics expressed in the Lagrangian, but also the one derived from the regularization procedure.

The NJL calculation retains some nice properties, in particular it preserves the sum rule and the polynomiality condition. Physically it is also very appealing since we can distinguish features associated with the weak and deep binding regimes. In Fig. 7 we show the variation of the quark distribution function with the binding energy. As the binding energy increases we change softly from a delta type behavior, at zero binding, to a constant behavior in the strong binding regime. This figure also illustrates nicely the phase transition associated with the spontaneous breaking of chiral symmetry. Suppose we keep the mass of the bound state fixed at $M^{2}=0$ and consider the variation of $q(x)$ with the constituent mass $m$. It is clear that we will always have $q(x)=1$ for any value of $m$, except when $m=0$, where the distribution function changes discontinuously to $q(x)=\delta(x-1 / 2)$. The effects of $t$ and $\zeta$ described 


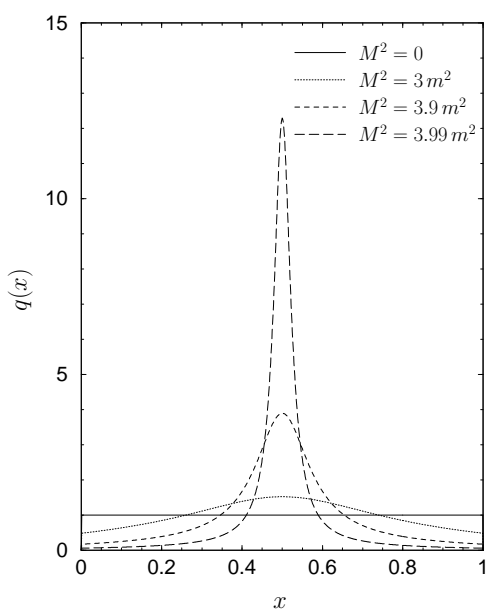

FIG. 7: Quark distribution function $q(x)=(x, 0,0)$ in the Nambu-Jona-Lasinio model. The constituent mass is kept fixed at $m=241 \mathrm{MeV}$.

for scalar Electrodynamics persist, as can be seen in the graph on the right hand side of this figure, i.e., $t$ pushes the distribution to higher values of $x$ and $\zeta$ introduces virtual pairs into the description of the system.

Unexpected results of the calculation are the non-vanishing of the GPDs at the boundary, i.e., $\mathcal{H}(x, \zeta, t) \neq 0$ for $x=1$ and $x=0$. This feature has nothing to do with what is usually called the support problem. The latter is characterized by a non-vanishing distribution function outside the physical region, i.e. $\mathcal{H}(x, \zeta, t) \neq 0$ for $x<\zeta-1$ or $x>1$, while in our calculations, the GPDs explicitly vanish there. It is therefore really a discontinuity that we encounter at the physical boundary.

For the quark parton distribution $q(x)=\mathcal{H}(x, 0,0)$, this peculiarity was already noted in refs. 32, 33, 34] and it is obviously present in an implicit way in the results of electromagnetic form factor calculations [15, 36, 37]. It showed up already in several model calculations $7,39,40,41,42$.

Another peculiarity of the calculation is the discontinuity at the $x=\zeta$. In the NJL model, the value of the GPD at $x=0$ and the discontinuity at $x=\zeta$ are related, as we have shown in Eq. (38), for any value of $M, t$ and $\zeta$. The value of $\mathcal{H}(0, \zeta, t)$ decreases as $t$ increases or as the binding energy decreases. But the origin of the non zero value of $\mathcal{H}(0, \zeta, t)$ is in the $\sigma$ diagrams depicted in Fig. 囵, which originate from the scalar four-fermion coupling present in the Lagrangian, Eq. (22).

Let us discuss the discontinuity at the boundaries further, within the NJL description. One might investigate the mass dependence of the GPDs at a given point $x$. In our calculation we get for $x=1$ the general expression

$$
\mathcal{H}(x=1, \zeta, t)=\frac{F_{0}}{F_{0}-1+\left(\sqrt{\frac{4 m^{2}}{M^{2}}-1}+\frac{1}{\sqrt{\frac{4 m^{2}}{M^{2}}-1}}\right) \arctan \left(\frac{1}{\sqrt{\frac{4 m^{2}}{M^{2}}-1}}\right)+\ldots},
$$

where $F_{0}=16 i \pi^{2} I_{2}(0)$, with $I_{2}(p)$ given by Eq. (B6) and the dots denote small terms of higher order in $M^{2} / \Lambda^{2}$, where $\Lambda$ is the cut-off parameter in the Pauli-Villars regularization, see appendix @ This equation illustrates the dependence on the bound state mass, and also the regularization scheme dependence through $F_{0}$ is apparent. It is clear from Eq. (50) that $\mathcal{H}(x=1, \zeta, t)$ vanishes for zero binding $\left(M^{2}=4 m^{2}\right)$ while it is non-vanishing, as soon as the interaction binds the quarks into a pion, i.e. $M^{2}<4 m^{2}$.

Moreover in the NJL model, for a small mass particle like the pion $\left(M=m_{\pi}\right)$ :

$$
\mathcal{H}(x=1, \zeta, t)=\frac{g_{\pi q q} f_{\pi}}{m_{\pi}}+\mathcal{O}\left(m_{\pi}\right)+\ldots=1+\mathcal{O}\left(m_{\pi}\right)+\ldots,
$$

where the last step follows from the Goldberger-Treiman relation as a consequence of spontaneously broken chiral symmetry, and to lowest order in $m_{\pi}$ this is regularization scheme independent.

The physical interpretation of these features is not completely clear at present. Let us however conjecture a solution based on an analysis of known results. The usual idea that the parton distributions must vanish on the boundary is based in the study of free parton models. If one of the partons carries all the + component of the momentum, i.e. $x=1$, the other one has $p^{+}=0$ and thus $p^{-}$goes to infinity if the particle is on shell. Since this $p^{-}$enters 
the propagator, the obvious conclusion is that the parton distribution must vanish at this boundary. But this simple description is broken when the particles are off-shell, like those in a bound system. Our result is therefore not unnatural and does not contradict any physical intuitive ideas. Moreover, the fact that the distributions do not vanish at $x=0$ for $\zeta=0$ is just a consequence of the non-vanishing at $x=1$ and does not seem to introduce any new conceptual problem.

One could suspect that this feature may originate as an artifact of the regularization in the NJL model, but this suspicion should be immediately dropped since it occurs in some limits in a regularization independent way. Therefore we conclude that there is a deeper physical reason for it, namely the off-shellness of the bound state quarks and the realization of chiral symmetry ${ }^{3}$.

Up to now we have been discussing the NJL model as a theory in itself. However the true theory of the strong interactions is QCD and the NJL model should be interpreted as an effective theory of QCD at low energies. QCD based arguments led in refs. 7, 39] to define a modified NJL model with a momentum dependent constituent quark mass (or equivalently, a constituent quark form factor) which, when appropriately chosen, makes the GPDs vanish at the end points. In ref. [42] the emphasis was placed in the perturbative aspects, i.e., gluons and sea bremsstrahlung. The QCD evolution equations 44] were used to eliminate the high momentum components. Certainly both mechanisms should be simultaneously advocated.

\section{CONCLUSION}

In this work, we presented a detailed calculation of generalized parton distribution functions using a covariant Bethe - Salpeter approach both in scalar Electrodynamics and in the Nambu-Jona-Lasinio model. No assumptions have gone into the determination of the Bethe - Salpeter amplitudes or any other ingredients of the calculation. The only approximations employed are the ladder approximation for the determination of the Bethe - Salpeter bound state amplitude (which is, of course, still a fully covariant object in this case), and in the determination of the current matrix elements, we have restricted ourselves to the lowest order diagrams (but we have shown that the next order correction vanishes exactly). As a result of this procedure, no important features required by general physical considerations, like symmetry properties, sum rules, etc., have been violated and we recover them in our numerical results. For the scalar model, the calculation evidences all desirable features, we reproduce the results obtained in similar studies, extend them to other kinematical regions and find sum rules which were not possible in the other treatments.

In the case of the NJL model, we found that the realization of chiral symmetry plays a crucial role in the outcome of the calculation. In the region $0<x<\zeta$ chiral symmetry intervenes explicitly through the contribution from the scalar channel. In the region $\zeta<x<1$, where the $\sigma$ does not contribute, it appears implicitly through the consistency equations which govern the dynamics of the pion and its coupling to quarks. In the massive case, this reflects in the fact that the GPDs do not vanish at the boundary of the kinematic region, i.e., at $x=0$ and $x=1$. Our detailed analysis associates this feature with the off-shellness of the constituent quarks, and the specific value there, with spontaneous chiral symmetry breaking. Moreover the GPDs are discontinuous at $x=\zeta$, a consequence of the $\sigma$ channel contribution,i.e., also a requirement of the chiral symmetry realization. These behaviors have been shown to arise in a regularization independent way.

In conclusion, we have shown that the SED model has continuous, well behaved GPDs that vanish at the end-points of the kinematic region, and bubble diagrams which do not contribute as a consequence of gauge symmetry. On the other hand, the NJL model has discontinuous, ill behaved GPDs, that are non-zero at the end-points, and bubble diagrams which, in the $\sigma$ channel, do contribute as a consequence of the realization of chiral symmetry.

Before finishing we must recall that our calculation is valid at the hadronic scale, i.e., at a low momentum renormalization point. Evolution to higher momenta is necessary to describe deep inelastic scattering data. It will be interesting to see how the described features of the NJL model will change under evolution. However the fact that the distributions do not vanish at the boundary imply the appearance of strong singularities which render the process non trivial.

\footnotetext{
${ }^{3}$ Similar features occur in the well defined 't Hooft model [43], a theoretical scheme which is exact in the large $N_{c}$ limit. In the chiral limit, this model produces a parton distribution $q(x)$ which is equal to 1 for any value of $x$, in a regularization independent way. But, when chiral symmetry is explicitly broken, the model of 't Hooft leads to a parton distribution which vanishes at the end-points, $q(x=0)=q(x=1)=0$. This different behavior is not surprising since the dynamics of this model and that of the NJL model are very different. The 't Hooft model is a $1+1$ dimensional model, which is confining, but without a point-like interaction.
} 


\section{Acknowledgments}

This work was supported by the European Commission IHP program under contract HPRN-CT-2000-00130, MCYT (Spain) under contracts BFM2001-3563-C02-01 and BMF2001-0262, and Generalitat Valenciana under contract GV01216. One of us (V.V.) would like to thank the Physics Department of Seoul National University, and Prof. Dong-Pil Min in particular, for the hospitality extended to him during the last stages of this work. We also want to thank the referees for their interesting comments which have improved the manuscript considerably.

\section{APPENDIX A: REGULARIZATION}

We have used the Pauli-Villars regularization in the Nambu-Jona-Lasinio model in order to render the occurring integrals finite. This means that for integrals like the ones defined by Eqs. (B1 B3), we make the replacement

$$
\int \frac{d^{4} p}{(2 \pi)^{4}} f\left(p ; m^{2}\right) \longrightarrow \int \frac{d^{4} p}{(2 \pi)^{4}} \sum_{j=0}^{2} c_{j} f\left(p ; m_{j}^{2}\right),
$$

with $m_{j}^{2}=m^{2}+j \Lambda^{2}, c_{0}=-c_{1} / 2=c_{2}=1$. Following ref. 15] we determine the regularization parameters $\Lambda$ and $m$ by calculating the pion decay constant and the quark condensate in the chiral limit via

$$
f_{\pi}^{2}=-\frac{3 m^{2}}{4 \pi^{2}} \sum_{j=0}^{2} c_{j} \log \left(m_{j}^{2} / m^{2}\right), \quad\langle\bar{u} u\rangle=-\frac{3 m}{4 \pi^{2}} \sum_{j=0}^{2} c_{j} m_{j}^{2} \log \left(m_{j}^{2} / m^{2}\right) .
$$

With the conventional values $\langle\bar{u} u\rangle=-(250 \mathrm{MeV})^{3}$ and $f_{\pi}=93 \mathrm{MeV}$, we get $m=241 \mathrm{MeV}$ and $\Lambda=859 \mathrm{MeV}$.

\section{APPENDIX B: ELEMENTARY INTEGRALS}

Some of the interesting integrals appearing in the main text are:

$$
\begin{gathered}
I_{1} \equiv \int \frac{d^{4} k}{(2 \pi)^{4}} \frac{1}{k^{2}-m^{2}+i \epsilon}, \\
I_{2}(p) \equiv \int \frac{d^{4} k}{(2 \pi)^{4}} \frac{1}{\left(k^{2}-m^{2}+i \epsilon\right)\left((k+p)^{2}-m^{2}+i \epsilon\right)}, \\
I_{3}\left(p_{1}, p_{2}\right) \equiv \int \frac{d^{4} k}{(2 \pi)^{4}} \frac{1}{\left(k^{2}-m^{2}+i \epsilon\right)\left(\left(k+p_{1}\right)^{2}-m^{2}+i \epsilon\right)\left(\left(k+p_{2}\right)^{2}-m^{2}+i \epsilon\right)},
\end{gathered}
$$

From these definitions the following relation may be deduced:

$$
\int \frac{d^{4} k}{(2 \pi)^{4}} \frac{k^{\mu}}{\left(k^{2}-m^{2}+i \epsilon\right)\left((k+p)^{2}-m^{2}+i \epsilon\right)}=-\frac{1}{2} p^{\mu} I_{2}(p) .
$$

The scalar model is renormalizable. The NJL model is not. We use in the latter case the Pauli-Villars regularization procedure and obtain:

$$
\begin{gathered}
I_{1}=-\frac{i}{16 \pi^{2}} \sum_{j=0}^{2} c_{j} m_{j}^{2} \log \left(m_{j}^{2} / m^{2}\right), \\
I_{2}(p)=-\frac{i}{16 \pi^{2}} \sum_{j=0}^{2} c_{j}\left\{\log \left(m_{j}^{2} / m^{2}\right)+\sqrt{\frac{p^{2}-4 m_{j}^{2}}{p^{2}}} \log \frac{1-\sqrt{\frac{p^{2}}{p^{2}-4 m_{j}^{2}}}}{1+\sqrt{\frac{p^{2}}{p^{2}-4 m_{j}^{2}}}}\right\} .
\end{gathered}
$$


From Eq. (B33) and $p_{1}^{2}=p_{2}^{2}=M^{2}$ we have:

$$
I_{3}\left(p_{1}, p_{2}\right)=I_{3}\left(t=\left(p_{1}-p_{2}\right)^{2}\right)=-\frac{i}{16 \pi^{2}} \sum_{j=0}^{2} c_{j} \int_{0}^{1} d z \frac{z}{\sqrt{d_{j}}} \ln \left|\frac{e_{j}+\sqrt{d_{j}}}{e_{j}-\sqrt{d_{j}}}\right|
$$

with

$$
d_{j}=t^{2} z^{4}-4 z^{2} t\left(m_{j}^{2}+M^{2} z(z-1)\right), \quad e_{j}=2 m_{j}^{2}+2 M^{2} z(z-1)-z^{2} t .
$$

The self consistency relation determining the constituent mass of the quark, $\mathrm{m}$, is

$$
m=\mu_{0}+8 i g N_{c} N_{f} m I_{1},
$$

while the equation for the mass of the pion, $P^{2}=M^{2}$, leads to

$$
1=4 i g N_{c} N_{f}\left[2 I_{1}-P^{2} I_{2}(P)\right],
$$

and the pion quark coupling constant, $g_{\pi q q}$, is given by

$$
g_{\pi q q}^{2}=\frac{-1}{12 i\left(I_{2}\left(M^{2}\right)+M^{2}\left(\partial I_{2}(p) / \partial p^{2}\right)_{p^{2}=M^{2}}\right)} .
$$

To obtain the results of Eqs. (113132), we used the following formulas:

$$
\begin{aligned}
& \tilde{I}_{2}(P) \equiv-16 i \pi^{2} \int \frac{d^{4} k}{(2 \pi)^{4}} \frac{\delta\left(x-k^{+} / P^{+}\right)}{\left(k^{2}-m^{2}+i \epsilon\right)\left((k-P)^{2}-m^{2}+i \epsilon\right)}=\left\{\begin{array}{cc}
\sum_{j=0}^{2} c_{j} \log \frac{m_{j}^{2}-\bar{x} P^{2}}{m^{2}}, & 0<x<1, \\
0 & \text { otherwise. }
\end{array}\right. \\
& \tilde{I}_{3}(m, x, \zeta, t) \equiv-16 i \pi^{2} \int \frac{d^{4} k}{(2 \pi)^{4}} \frac{\delta\left(x-k^{+} / P^{+}\right)}{\left(k^{2}-m^{2}+i \epsilon\right)\left((k+\Delta)^{2}-m^{2}+i \epsilon\right)\left((P-k)^{2}-m^{2}+i \epsilon\right)}= \\
&=\left\{\begin{array}{cc}
\frac{1}{\sqrt{D}} \log \frac{[2(1-\zeta)(\zeta y-x)-\zeta] M^{2}-(1-x) t+2 m^{2} / y+\sqrt{D}}{[2(1-\zeta)(\zeta y-x)-\zeta] M^{2}-(1-x) t+2 m^{2} / y-\sqrt{D}}, & 0<x<1, \\
0 & \text { otherwise. }
\end{array}\right.
\end{aligned}
$$

In the last integral, $D=\zeta^{2} M^{2}\left(M^{2}-4 m^{2}\right)+(1-x)^{2} t^{2}+2(1-x)(2 x-\zeta) M^{2} t-4 m^{2}(1-\zeta) t, \zeta=-\Delta^{+} / P^{+}, M^{2}=P^{2}$, $t=\Delta^{2}$ and

$$
y=\left\{\begin{array}{cc}
x / \zeta, & 0<x<\zeta \\
(1-x) /(1-\zeta), & \zeta<x<1
\end{array}\right.
$$

\section{APPENDIX C: CONTRIBUTION OF THE $\sigma$ - DIAGRAMS}

The contribution of the diagrams shown in Fig. [5 can be calculated as $\mathcal{H}^{b}(x, \zeta, t)=A \cdot B$, with

$$
A=\frac{1}{2} \int \frac{d^{4} p}{(2 \pi)^{4}} \delta\left(x-\frac{p^{+}}{P^{+}}\right) \operatorname{Tr}\left[i S(p) \gamma^{+} \frac{1}{2}\left(1+\tau^{3}\right) i S(p+\Delta)\right]
$$

and

$$
B=\frac{2 g_{\pi q q}^{2} g}{1-2 g \Pi_{s}\left(P-P^{\prime}\right)} \int \frac{d^{4} k}{(2 \pi)^{4}} \operatorname{Tr}\left[\gamma_{5} \tau^{-} S(k-P) \gamma_{5} \tau^{+} S(k+\Delta) S(k)+\gamma_{5} \tau^{+} S\left(k+P^{\prime}\right) \gamma_{5} \tau^{-} S(k+\Delta) S(k)\right],
$$


where $\Pi_{s}$ is the scalar vacuum polarization:

$$
\Pi_{s}(P)=-i \int \frac{d^{4} p}{(2 \pi)^{4}} \operatorname{Tr}[i S(p) i S(p-P)]=4 i N_{c} N_{f}\left[I_{1}+\frac{1}{2}\left(4 m^{2}-P^{2}\right) I_{2}(P)\right] .
$$

After some algebra we obtain using Eq. (B10)

$$
\mathcal{H}^{b}(x, \zeta, t)=\frac{1}{4 \pi^{2}} N_{c} g_{\pi q q}^{2} \theta(\zeta-x) \theta(x)\left(1-\frac{2 x}{\zeta}\right) C(t) \sum_{j=1}^{2} c_{j}\left[-\ln \frac{m_{j}^{2}}{m^{2}}-\ln \frac{m_{j}^{2}-t \bar{y}}{m_{j}^{2}}\right]
$$

with $\bar{y}=x(\zeta-x) / \zeta^{2}$ and

$$
C(t)=m^{2} \frac{\left[\left(t-2 M^{2}\right) I_{3}\left(P, P^{\prime}\right)+2 I_{2}\left(P-P^{\prime}\right)\right]}{M^{2} I_{2}\left(M^{2}\right)+\left(4 m^{2}-t\right) I_{2}\left(P-P^{\prime}\right)}
$$

which are Eqs. (33) and (34).

\section{APPENDIX D: POLYNOMIALITY.}

One stringent test of models is the polynomiality condition. This study appears simpler and more physical in terms of the more symmetric variables [4]:

$$
X=\frac{x-\zeta / 2}{1-\zeta / 2}, \quad \xi=\frac{\zeta}{2-\zeta} .
$$

With this definition $X$ ranges from -1 to +1 , and not from $\zeta-1$ to +1 , which is the range associated with $x$. In terms of these new variables the polynomiality condition is stated as [4]:

$$
H_{n}(\xi, t)=\int_{-1}^{+1} d X X^{n-1} \mathcal{H}(X, \xi, t)=\sum_{i=0}^{\left[\frac{n}{2}\right]} A_{n, 2 i}(t) \xi^{2 i}
$$

where $[\ldots]$ means the integer part. Time reversal invariance and hermiticity imply that the distributions $H_{n}(\xi, t)$ are even functions of $\xi[4]$.

For SED in the case $m_{\pi}=0$ and $t=0$, as function of the new variables, we have

$$
\mathcal{H}(X, \xi, 0)=\left\{\begin{array}{lc}
\frac{6}{1+\xi} \frac{X(X+\xi)}{2 \xi}, & -\xi<X<\xi \\
\frac{6}{1+\xi} \frac{X(1-X)}{1-\xi}, & \xi<X<1
\end{array}\right.
$$

Using Eq. (D2) we obtain for the coefficients,

$$
A_{n, 2 i}^{S E D}(0)=\frac{6}{(n+1)(n+2)} .
$$

This simple closed form only appears for vanishing $t$ and $m_{\pi}$. For non zero values we have to proceed numerically. In table \we show values of $A_{n, 2 i}^{S E D}(t)$ for the lowest values of $n$, and non vanishing $t$ and $m_{\pi}$. These results show that $A_{n, 2 i}^{S E D}(t)$ are not anymore independent of $i$. We also obtain that the effect of a physical pion mass is small, i.e. at the level of a few percent.

The definition of the coefficients in Eq. (D4) leads to the following relation for their sum, which is connected with the value of the moments (D2) at $\xi=1$,

$$
H_{2 m-1}(1,0)=H_{2 m}(1,0),
$$

with $m=1,2, \ldots$ We have verified numerically (with a precision of $10^{-13}$ ) that this relation is also exactly satisfied for $t \neq 0$ and $m_{\pi}=0$ :

$$
H_{2 m-1}(1, t)=\sum_{i=0}^{m-1} A_{2 m-1,2 i}^{S E D}(t)=H_{2 m}(1, t)=\sum_{i=0}^{m} A_{2 m, 2 i}^{S E D}(t)
$$


For $m_{\pi} \neq 0$ the maximum value of $\xi$ never reaches 1 and therefore we cannot connect the sum of the $A_{n, 2 i}$ coefficients with the value of the moment at any physical value of $\xi$. Our numerical study shows however that, in the case of SED, we have an approximate sum rule for the sum of coefficients $A_{n, 2 i}$ :

$$
\sum_{i=0}^{m-1} A_{2 m-1,2 i}^{S E D}(t)-\sum_{i=0}^{m} A_{2 m, 2 i}^{S E D}(t) \simeq \mathcal{O}\left(m_{\pi}^{2}\right)
$$

The deviation from the exact sum rule is at the level of one percent for $t=-10^{-5} \mathrm{GeV}^{2}(-t$ small) and at the level of $10^{-3}$ for $t=-10 \mathrm{GeV}^{2}$ (-t large).

We now turn to the NJL model. As in the SED case, we start from the parton distribution for $t=0$ and $m_{\pi}=0$ :

$$
\mathcal{H}(X, \xi, 0)=\frac{1}{2} \theta(\xi-X) \theta(\xi+X)+\theta(X-\xi) \theta(1-X)
$$

Inserting $\mathrm{Eq}(\mathrm{D} 8)$ in Eq. (D2) and integrating over $X$ we obtain the coefficients,

$$
\begin{aligned}
& A_{n, 0}^{N J L}(0)=\frac{1}{n}, \\
& A_{n, 2 i}^{N J L}(0)=0, \quad 1 \leq 2 i \leq n-1, \\
& A_{n, n}^{N J L}(0)=\frac{-1}{n} \delta_{\left[\frac{n}{2}\right], \frac{n}{2}} .
\end{aligned}
$$

In this case, the closed forms appear only for vanishing values of $m_{\pi}$ and $t$. Results for $t \neq 0$ and $m_{\pi} \neq 0$ have been obtained numerically and are shown in table \. From these results we realize that $A_{n, 2 i}^{N J L}(t)$ are not zero for $1 \leq 2 i \leq n-1$. We also observe that the effect of a physical pion mass is small.

We have found also several simple relations like

$$
H_{2 m}(1,0)=0, \quad H_{2 m+1}(1,0)=H_{2 m+1}(0,0),
$$

with $m=1,2 \ldots$. But in this case these relations are only approximately satisfied for $t \neq 0$ and $m_{\pi}=0$ or for $m_{\pi} \neq 0$.

We have studied numerically the contributions to $H_{n}(\xi, t)$ coming from each diagram separately. We observe that there are independent sum rules for each diagram but they disappear when the sum is performed. The contribution coming from the diagram in Fig. 4 verifies the relation

$$
\sum_{i=0}^{m} A_{2 m, 2 i}^{N J L}(t)-\sum_{i=0}^{m} A_{2 m+1,2 i}^{N J L}(t)=\mathcal{O}\left(m_{\pi}^{2}\right)
$$

exactly for $m=0$ and any $t$, and in an approximate way (deviations less than $10^{-3}$ ) for $m_{\pi}=140 \mathrm{MeV}$. On the other hand, the diagram in Fig. 5 gives non-vanishing contributions only to the coefficients $A_{n, n}^{N J L}$ for any value of $m_{\pi}$ and any value of $t$.

Regarding the numerical values of these coefficients we observe that in the scalar model and for large $n, H_{n}(0,0)$ decreases with $n$ as $1 / n^{2}$ whereas $H_{n}(1,0)$ decreases with $n$ as $1 / n$, showing that $\mathcal{H}(X, 1,0)$ is more concentrated around $X=1$ than $\mathcal{H}(X, 0,0)$. In the NJL case for large $n, H_{n}(0,0)$ and $H_{n}(1,0)$ decrease as $1 / n$. This shows that $\mathcal{H}(X, \xi, 0)$ is more concentrated around the $X=1$ region than in the scalar case. These asymptotic behaviors appear for any value of $t$ and $m_{\pi}$.

The numerical analysis also shows that for small $n$, as $-t$ increases, the coefficients of the SED and NJL models are of the same order, loosing the characteristic behavior of equations (D4) and (D9). Nevertheless we observe a regularity in the signs: all the coefficients are positive in the scalar model, whereas in the NJL model the coefficients $A_{n, 0}$ are positive but the rest are negative, except some small coefficients present at small $-t$.

Finally we recall that $A_{1,0}(0)=1$ corresponds to the charge sum rule and $A_{2,0}(0)=1 / 2$ is related to the momentum sum rule.

[1] D. Müller, D. Robaschik, B. Geyer, F. M. Dittes, and J. Horejsi, Fortschr. Phys. 42, 101 (1994).

[2] X.-D. Ji, Phys. Rev. D55, 7114 (1997).

[3] A. V. Radyushkin, Phys. Rev. D56, 5524 (1997).

[4] X.-D. Ji, J. Phys. G24, 1181 (1998). 


\begin{tabular}{|c|c|c|c|c|c|c|c|c|c|}
\hline \multicolumn{2}{|c|}{$n$} & 1 & \multicolumn{2}{|r|}{2} & \multicolumn{2}{|c|}{3} & \multicolumn{3}{|c|}{4} \\
\hline & & $A_{1,0}(t)$ & $A_{2,0}(t)$ & $A_{2,2}(t)$ & $A_{3,0}(t)$ & $A_{3,2}(t)$ & $A_{4,0}(t)$ & $A_{4,2}(t)$ & $A_{4,4}(t)$ \\
\hline \multirow{3}{*}{$\begin{array}{c}\text { SED } \\
m_{\pi}=0\end{array}$} & $t=0$ & 1. & 0.5 & 0.5 & 0.3 & 0.3 & 0.2 & 0.2 & 0.2 \\
\hline & $t=-1$ & 0.629 & 0.355 & 0.274 & 0.230 & 0.187 & 0.162 & 0.137 & 0.119 \\
\hline & $t=-10$ & 0.240 & 0.157 & 0.083 & 0.113 & 0.065 & 0.087 & 0.053 & 0.039 \\
\hline \multirow{3}{*}{$\begin{array}{c}\text { SED } \\
m_{\pi}=140\end{array}$} & $t=0\left[-10^{-5}\right]$ & 1. & 0.5 & [0.488] & 0.299 & {$[0.299]$} & 0.199 & {$[0.201]$} & {$[0.166]$} \\
\hline & $t=-1$ & 0.615 & 0.348 & 0.264 & 0.226 & 0.183 & 0.158 & 0.135 & 0.114 \\
\hline & $t=-10$ & 0.229 & 0.150 & 0.079 & 0.109 & 0.063 & 0.083 & 0.051 & 0.037 \\
\hline \multirow{3}{*}{$\begin{array}{c}\text { NJL } \\
m_{\pi}=0\end{array}$} & $t=0$ & 1. & 0.5 & -0.5 & 0.333 & 0. & 0.25 & 0. & -0.25 \\
\hline & $t=-1$ & 0.487 & 0.336 & -0.240 & 0.257 & -0.049 & 0.208 & -0.030 & -0.113 \\
\hline & $t=-10$ & 0.091 & 0.119 & -0.093 & 0.118 & -0.058 & 0.111 & -0.046 & -0.044 \\
\hline \multirow{3}{*}{$\begin{array}{c}\text { NJL } \\
m_{\pi}=140\end{array}$} & $t=0\left[-10^{-5}\right]$ & 1. & 0.5 & {$[-0.473]$} & 0.332 & {$[0.005]$} & 0.247 & {$[0.005]$} & {$[-0.236]$} \\
\hline & $t=-1$ & 0.482 & 0.332 & -0.230 & 0.253 & -0.045 & 0.203 & -0.026 & -0.109 \\
\hline & $t=-10$ & 0.090 & 0.116 & -0.088 & 0.114 & -0.055 & 0.108 & -0.043 & -0.042 \\
\hline
\end{tabular}

TABLE I: Coefficients of the polynomial expansion. The pion mass is expressed in MeV and $t$ is expressed in GeV ${ }^{2}$ Values between brackets correspond to $t=-10^{-5} \mathrm{GeV}^{2}$.

[5] M. Diehl, T. Feldmann, R. Jakob, and P. Kroll, Eur. Phys. J. C8, 409 (1999).

[6] M. Diehl, T. Feldmann, R. Jakob, and P. Kroll, Nucl. Phys. B596, 33 (2001).

[7] M. V. Polyakov and C. Weiss, Phys. Rev. D60, 114017 (1999).

[8] M. Diehl, T. Feldmann, P. Kroll, and C. Vogt, Phys. Rev. D61, 074029 (2000).

[9] X.-D. Ji, W. Melnitchouk, and X. Song, Phys. Rev. D56, 5511 (1997).

[10] I. V. Anikin, D. Binosi, R. Medrano, S. Noguera, and V. Vento, Eur. Phys. J. A14, 95 (2002).

[11] V. Y. Petrov et al., Phys. Rev. D57, 4325 (1998).

[12] M. Penttinen, M. V. Polyakov, and K. Goeke, Phys. Rev. D62, 014024 (2000).

[13] S. Scopetta and V. Vento, Nucl. Phys. A711, 190 (2002).

[14] S. Scopetta and V. Vento, Eur. Phys. J. A16, 527 (2003).

[15] S. P. Klevansky, Rev. Mod. Phys. 64, 649 (1992).

[16] J. Bijnens, Phys. Rept. 265, 369 (1996).

[17] K. J. Golec-Biernat and A. D. Martin, Phys. Rev. D59, 014029 (1999).

[18] P. A. M. Guichon and M. Vanderhaeghen, Prog. Part. Nucl. Phys. 41, 125 (1998).

[19] C. Itzykson and J. B. Zuber, Quantum Field Theory (McGraw-Hill, 1985).

[20] B. Desplanques, L. Theußl, and S. Noguera, Phys. Rev. C65, 038202 (2002).

[21] A. Amghar, B. Desplanques, and L. Theußl, Nucl. Phys. A714, 213 (2003).

[22] B. C. Tiburzi and G. A. Miller, Phys. Rev. D67, 113004 (2003).

[23] B. C. Tiburzi and G. A. Miller, Phys. Rev. C64, 065204 (2001).

[24] Y. Nambu and G. Jona-Lasinio, Phys. Rev. 122, 345 (1961).

[25] Y. Nambu and G. Jona-Lasinio, Phys. Rev. 124, 246 (1961).

[26] U. Vogl and W. Weise, Prog. Part. Nucl. Phys. 27, 195 (1991).

[27] I. V. Anikin, A. E. Dorokhov, A. E. Maksimov, L. Tomio, and V. Vento, Nucl. Phys. A678, 175 (2000).

[28] C. Vogt, Phys. Rev. D64, 057501 (2001).

[29] H.-M. Choi, C.-R. Ji, and L. S. Kisslinger, Phys. Rev. D64, 093006 (2001).

[30] L. S. Kisslinger, H.-M. Choi, and C.-R. Ji, Phys. Rev. D63, 113005 (2001).

[31] R. M. Davidson and E. Ruiz Arriola, Acta Phys. Polon. B33, 1791 (2002).

[32] R. M. Davidson and E. Ruiz Arriola, Phys. Lett. B348, 163 (1995).

[33] H. Weigel, E. Ruiz Arriola, and L. P. Gamberg, Nucl. Phys. B560, 383 (1999).

[34] T. Shigetani, K. Suzuki, and H. Toki, Phys. Lett. B308, 383 (1993).

[35] M. V. Polyakov, Nucl. Phys. B555, 231 (1999).

[36] V. Bernard and U. G. Meissner, Phys. Rev. Lett. 61, 2296 (1988).

[37] H. J. Schulze, J. Phys. G20, 531 (1994).

[38] B. C. Tiburzi and G. A. Miller, Phys. Rev. D65, 074009 (2002).

[39] M. Praszalowicz and A. Rostworowski (2002), hep-ph/0205177.

[40] A. E. Dorokhov and L. Tomio, Phys. Rev. D62, 014016 (2000).

[41] I. V. Anikin, A. E. Dorokhov, and L. Tomio, Phys. Lett. B475, 361 (2000).

[42] E. Ruiz Arriola and W. Broniowski, Phys. Rev. D66, 094016 (2002).

[43] G. 't Hooft, Nucl. Phys. B75, 461 (1974).

[44] D. Müller, Phys. Rev. D51, 3855 (1995). 